\title{
Sophorolipid protects against early-weaning syndrome by improving the gut microenvironment in early-weaned piglets
}

Min-jin Kwak ( $\sim$ ics1051@korea.ac.kr)

Korea University - Seoul Campus: Korea University https://orcid.org/0000-0001-9832-3251

\section{Sun-Woo Choi}

Korea University - Seoul Campus: Korea University

\section{Yong-Soon Choi}

Korea University - Seoul Campus: Korea University

\section{Hanbae Lee}

Pathway Intermediates

Kwang-Youn Whang

Korea University - Seoul Campus: Korea University

\section{Research Article}

Keywords: Pig, gut microbiota, gut morphology, local inflammation, mucus, sophorolipids

Posted Date: September 17th, 2021

DOl: https://doi.org/10.21203/rs.3.rs-910531/v1

License: (c) (1) This work is licensed under a Creative Commons Attribution 4.0 International License. Read Full License

Version of Record: A version of this preprint was published at BMC Veterinary Research on January 3rd, 2022. See the published version at https://doi.org/10.1186/s12917-021-03105-3. 


\section{Abstract \\ Background}

In animals, weaning stress is the first and most critical stress. Weaning can negatively affect the growth performance of animals physically, psychologically, and pathologically. Our previous studies on the HT-29 cell line and early-weaned rats demonstrated that adequate sophorolipid (SPL) supplementation in feed could enhance the mucin-producing and wound healing capacities of the gut defense system by modulating gut microbiota.

\section{Results}

Dietary SPL supplementation at 5 and $10 \mathrm{mg} / \mathrm{kg}$ quadratically increased the average daily gain during the experimental period in the treatment groups when compared with the control group. The albumin levels of piglets fed with the SPL supplemented diet were downregulated to the normal range. Moreover, in feed, SPL supplementation at 5 and $10 \mathrm{mg} / \mathrm{kg}$ improved jejunal histological indices and gene expression levels related to mucin secretion and local inflammation markers. Consistent with these results, adequate SPL supplementation ( 5 and $10 \mathrm{mg} / \mathrm{kg}$ ) increased the population of Lactobacillus, a beneficial bacteria, and its short-chain fatty acid production in the ceca of piglets.

\section{Conclusions}

The occurrence of diarrhea after weaning in piglets could be reduced by feeding an SPL-supplemented diet which improves the gut defense system by increasing the microbial population and enhancing mucin layer integrity.

\section{Background}

Weaning is the transitional phase of feeding in mammals from reliance on the mother's milk to an adult diet. This process is stressful for mammals because changing feed form could result in wounds in the digestive tract, especially in the villi [1]. Neuenschwander et al. [2] demonstrated that weaning significantly decreased growth performance and nutrient digestibility. To solve these problems, the use of sub-therapeutic dosages of antibiotic growth promoters has been widely adopted in the livestock industry owing to their outstanding efficacy in feed conversion and animal growth [3]. Nonetheless, antibiotic growth promoters have been banned because of the increasing risk of the microbial resistome [4]. Therefore, the livestock feed industry seeks alternatives to antibiotics, including organic and inorganic acids, enzymes, pro- and prebiotics, phytochemicals, and nano-compounds [5-6]

Consequently, the development of novel and eco-friendly materials (e.g., probiotics, prebiotics, organic acids, essential oils, and enzymes) to replace antibiotic growth promoters is necessary [7]. Additionally, 
various bio-surfactants have been investigated because of their antibacterial properties [8]. Among biosurfactants, sophorolipids (SPLs) have received much attention in various fields, such as medicine, hygiene, and pharmaco-dermatology, owing to their relatively low toxicity and biodegradability [9].

SPLs are a type of glycolipid bio-surfactant mainly produced by several non-pathogenic yeast species, such as Candida bombicola. They are composed of a dimeric sugar linked by a glycosidic bond to a hydroxyl fatty acid [10]. SPLs are less toxic and more biodegradable than other known surfactants [1112] and display various unique biological properties, including immunomodulation, depigmentation, skin dermal fibroblast stimulation, and collagen production [13]. Additionally, SPLs exhibit antimicrobial, anticancer, antiviral, and antifungal properties as well as cytotoxicity [14-15]. These properties imply that SPLs can be applied in fields such as medicine, hygiene, and pharmaco-dermatology. However, despite their considerable potential, the use of SPLs in the animal feed industry has not yet been reported.

Therefore, the present study aimed to determine the effects of dietary SPL supplementation on the gastrointestinal health in weaned piglets. To this effect, we conducted a dietary experiment with earlyweaned piglets a concentration of 5,10 , and $15 \mathrm{mg} / \mathrm{kg}$ according to previous dosage-determining experiments in piglets.

\section{Materials And Methods Piglets and diets}

We conducted all animal studies in accordance with the guidelines and regulations of the Animal Ethics Committee approved by Korea University (Seoul, Republic of Korea). The experiment was performed at a clean, controlled research farm at Dankook University in Cheonan, Republic of Korea. A total of 140 earlyweaned piglets ( $L \times Y \times D ; 21$ days old) were randomly allotted to four treatments according to their body weight (BW; initial BW: $6.57 \mathrm{~kg}$ ). Each treatment was replicated seven times, and there were five piglets in each pen (each pen being an experimental unit). The dietary treatments consisted of CON (basal diet), SPL5 (5 mg/kg SPL supplementation), SPL10 (10 mg/kg SPL supplementation), and SPL15 (15 mg/kg SPL supplementation). The feed composition and calculated nutritional values are shown in Table 1. The feed and SPL were supplied by EASY BIO Inc. (Seoul, Republic of Korea). Feed and water were supplied ad libitum to the pigs during the experiment. The raising program consisted of three phases: phase 1, day 1-7; phase 2, day 8-21; phase 3, day 22-42. The BW and feed intake were measured biweekly to calculate average daily gain (ADG), average daily feed intake (ADFI), and feed efficiency (FE). 
Table 1

Composition and nutritional value of basal diets ${ }^{1}$

\begin{tabular}{|llll|}
\hline Ingredients, \% & Phase 1 & Phase 2 & Phase 3 \\
\hline Corn & 47.80 & 53.90 & 62.40 \\
\hline Soybean meal & 5.00 & 20.15 & 24.20 \\
\hline Fish meal & 4.50 & 4.00 & 3.00 \\
\hline Plasma powder & 5.00 & 2.50 & 0.00 \\
\hline Whey powder & 20.00 & 10.00 & 5.00 \\
\hline Lacotse & 8.00 & 3.00 & 0.00 \\
\hline Soybean oil & 5.50 & 2.50 & 2.00 \\
\hline Lysine sulfate & 0.38 & 0.46 & 0.32 \\
\hline Methionine & 0.31 & 0.24 & 0.16 \\
\hline Threonine & 0.15 & 0.15 & 0.10 \\
\hline Tryptophane & 0.05 & 0.05 & 0.02 \\
\hline Choline chloride & 0.10 & 0.05 & 0.05 \\
\hline MCP & 0.86 & 1.17 & 0.60 \\
\hline Limestone & 1.52 & 1.00 & 1.33 \\
\hline Salt & 0.20 & 0.20 & 0.20 \\
\hline Zinc oxide & 0.28 & 0.28 & 0.28 \\
\hline Vitamin premix & 0.12 & 0.12 & 0.12 \\
\hline Mineral premix & 0.15 & 0.05 & 0.05 \\
\hline Phytase & 0.05 & & 0.15 \\
\hline NSPase & & 0.03 & \\
\hline
\end{tabular}

1Abbreviations:

$\mathrm{CP}$, crude protein; MCP, monocalcium phosphate; ME, metabolizable energy; NSPase, non-starch polysaccharidase.

2Provided per kilogram of complete diet: vitamin A, 12,000 IU; vitamin $\mathrm{D}_{3}, 2,500 \mathrm{IU}$; vitamin $\mathrm{E}, 30 \mathrm{IU}$; vitamin $\mathrm{K}_{3}, 3 \mathrm{mg}$; pantothenic acid, $15 \mathrm{mg}$; nicotinic acid, 40 mg; choline, $400 \mathrm{mg}$; vitamin $\mathrm{B}_{12}, 12 \mu \mathrm{g}$

${ }^{3}$ Provided per kilogram of complete diet: iron, $90 \mathrm{mg}$; copper, $8.8 \mathrm{mg}$; zinc, $100 \mathrm{mg}$; manganese, 54 $\mathrm{mg}$; iodine, $0.35 \mathrm{mg}$; selenium, $0.30 \mathrm{mg}$. 


\begin{tabular}{|c|c|c|c|}
\hline Ingredients, \% & Phase 1 & Phase 2 & Phase 3 \\
\hline Total & 100.00 & 100.00 & 100.00 \\
\hline \multicolumn{4}{|l|}{ Calculated value } \\
\hline ME (kcal/ kg) & 3673.00 & 3544.00 & 3467.00 \\
\hline CP (\%) & 20.63 & 19.80 & 18.80 \\
\hline Calcium (\%) & 0.98 & 0.75 & 0.75 \\
\hline Phosphate (\%) & 0.65 & 0.68 & 0.50 \\
\hline Lysine (\%) & 1.60 & 1.41 & 1.22 \\
\hline Methionine (\%) & 0.62 & 0.55 & 0.46 \\
\hline Threonine (\%) & 1.07 & 0.95 & 0.83 \\
\hline Tryptophan (\%) & 0.32 & 0.29 & 0.24 \\
\hline \multicolumn{4}{|l|}{ 1Abbreviations: } \\
\hline \multicolumn{4}{|c|}{$\begin{array}{l}\text { CP, crude protein; } \mathrm{MCP} \text {, monocalcium phosphate; ME, metabolizable energy; NSPase, non-starch } \\
\text { polysaccharidase. }\end{array}$} \\
\hline \multicolumn{4}{|c|}{$\begin{array}{l}{ }^{2} \text { Provided per kilogram of complete diet: vitamin A, 12,000 IU; vitamin } D_{3}, 2,500 \mathrm{IU} \text {; vitamin } \mathrm{E}, 30 \mathrm{IU} \text {; } \\
\text { vitamin } \mathrm{K}_{3}, 3 \mathrm{mg} \text {; pantothenic acid, } 15 \mathrm{mg} \text {; nicotinic acid, } 40 \mathrm{mg} \text {; choline, } 400 \mathrm{mg} \text {; vitamin } \mathrm{B}_{12}, 12 \mu \mathrm{g}\end{array}$} \\
\hline \multicolumn{4}{|c|}{$\begin{array}{l}{ }^{3} \text { Provided per kilogram of complete diet: iron, } 90 \text { mg; copper, } 8.8 \mathrm{mg} \text {; zinc, } 100 \text { mg; manganese, } 54 \\
\text { mg; iodine, } 0.35 \text { mg; selenium, } 0.30 \mathrm{mg} \text {. }\end{array}$} \\
\hline
\end{tabular}


Table 3

Growth performance of weaning pigs fed experimental diets

\begin{tabular}{|c|c|c|}
\hline Gene name & Sequence (forward, reverse) & Reference \\
\hline \multicolumn{3}{|c|}{ Housekeeping gene } \\
\hline \multirow[t]{2}{*}{ GAPDH } & F: 5'- GAGGTCGGAGTGAACGGAT - 3' & \multirow[t]{2}{*}{ [38] } \\
\hline & R: 5'- CCTGGGTCGAATCATACTGGAACA - 3' & \\
\hline \multicolumn{3}{|c|}{ Inflammatory cytokines } \\
\hline \multirow[t]{2}{*}{ IL-8 } & F: 5'- TTTCTGCAGCTCTCTGTGAGG - 3' & \multirow[t]{2}{*}{ [39] } \\
\hline & R: 5'- CTGCTGTTGTTGTTGCTTCTC - 3' & \\
\hline \multirow[t]{2}{*}{ IFN-y } & F: 5'- GTTTTTCTGGCTCTTACTGC - 3' & \multirow[t]{2}{*}{ [40] } \\
\hline & R: 5'- CTTCCGCTTTCTTAGGTTAG - 3' & \\
\hline \multirow[t]{2}{*}{ TNF-a } & F: 5'- ATCGGCCCCCAGAAGGAAGAG - 3' & \multirow[t]{2}{*}{ [41] } \\
\hline & R: 5'- GATGGCAGAGAGGAGGTTGAC - 3' & \\
\hline \multirow[t]{2}{*}{ IL-10 } & F: 5'- GCATCCACTTCCCAACCA - 3' & \multirow[t]{2}{*}{ [42] } \\
\hline & R: 5'- CTTCCTCATCTTCATCGTCAT - 3' & \\
\hline \multicolumn{3}{|c|}{ Tight junction proteins } \\
\hline \multirow[t]{2}{*}{ ZO-1 } & F: 5'- AAGCCCTAAGTTCAATCACAATCT - 3' & \multirow[t]{2}{*}{ [43] } \\
\hline & R: 5'- ATCAAACTCAGGAGGCGGC - 3' & \\
\hline \multirow[t]{2}{*}{ OCLD } & F: 5'- TCCTGGGTGTGATGGTGTTC - 3' & \multirow[t]{2}{*}{ [43] } \\
\hline & R: 5'- CGTAGAGTCCAGTCACCGCA - 3' & \\
\hline \multirow[t]{2}{*}{ CLDN1 } & F: 5'- TCGACTCCTTGCTGAATCTG - 3' & \multirow[t]{2}{*}{ [38] } \\
\hline & R: 5'- TTACCATACCTTGCTGTGGC - 3' & \\
\hline \multicolumn{3}{|c|}{ Mucin production } \\
\hline \multirow[t]{2}{*}{ MUC2 } & F: 5'- GGCTGCTCATTGAGAGGAGT - 3' & \multirow[t]{2}{*}{ [44] } \\
\hline & R: 5'- ATGTTCCCGAACTCCAAGG - 3' & \\
\hline
\end{tabular}




\begin{tabular}{|lllllllll|}
\hline \multicolumn{3}{|c}{ Dietary SPL levels (ppm) } & \multicolumn{5}{c|}{ Polynomial contrast $^{2}$} \\
\hline \multicolumn{1}{|c}{0} & 5 & 10 & 15 & SEM $^{1}$ & L & Q & C \\
\hline Body weight, kg & & & & & & & \\
\hline Week 0 & 6.56 & 6.58 & 6.60 & 6.57 & 0.24 & 0.990 & 0.978 & 0.970 \\
\hline Week 2 & 10.16 & 10.15 & 10.19 & 10.08 & 0.25 & 0.918 & 0.924 & 0.908 \\
\hline Week 4 & 16.34 & 16.49 & 16.51 & 16.27 & 0.26 & 0.926 & 0.718 & 0.945 \\
\hline Week 6 & 24.97 & 25.43 & 25.26 & 24.82 & 0.28 & 0.825 & 0.465 & 0.901 \\
\hline Overall & & & & & & & & \\
\hline ADG, g & 438.26 & 448.91 & 444.21 & 434.39 & 2.55 & 0.446 & 0.048 & 0.621 \\
\hline ADFl, g & 598.14 & 602.64 & 589.87 & 591.21 & 4.36 & 0.410 & 0.881 & 0.430 \\
\hline FE & 0.733 & 0.746 & 0.754 & 0.736 & 0.006 & 0.777 & 0.219 & 0.684 \\
\hline 1 Standard error of mean. & & & & & & \\
\hline 2 L, linear; Q, quadratic; C, cubic & & & & & & & \\
\hline
\end{tabular}

\section{Sample Collection}

At the end of the experiment, 21 piglets (one piglet per pen, randomly selected) were sacrificed, and blood samples were collected from the jugular vein into heparin-coated plasma tubes (BD Vacutainer; Beckton Dickinson Rowa Denmark, Kongens Lyngby, Denmark) for analysis of biochemical markers (glucose, triglyceride, total cholesterol, blood urea nitrogen, albumin, and creatinine). Thereafter, jejunal and cecal samples were obtained, immediately frozen using dry ice, and stored at $-80^{\circ} \mathrm{C}$ until further analysis. In addition, parts of the jejunum were fixed in a $4 \%$ formalin solution for histological analysis.

\section{Blood biochemical markers}

The concentrations of glucose, triglyceride, total cholesterol, blood urea nitrogen, albumin, and creatinine in the plasma samples were determined using commercial kits (EMBIELTM, Seoul, Korea) according to the manufacturer's instructions. The absorbance of samples was measured using a spectrophotometer (Zenyth 200rt; Biochrom, Berlin, Germany) at a specific wavelength, and the concentration of samples was calculated using the standard curve of each biomarker.

\section{Histological analysis of the jejunum}

The fixed jejunum samples were embedded into paraffin blocks, and $5 \mu \mathrm{m}$ cross-sections were prepared using a rotary microtome (CUT 5062; SLEE Medical, Mainz, Germany). The jejunum sections were then stained with hematoxylin and eosin and Alcian blue. Subsequently, a total of 15 villi and 15 crypts were 
randomly selected per experimental unit, and a single observer measured the villus height and crypt depth, and counted the number of goblet cells.

\section{RNA extraction from the jejunum and cecum}

Total RNA from jejunal and cecal samples were extracted using Trizol® (Invitrogen, Grand Island, NY, USA) according to the manufacturer's instructions, and the concentration and purity of RNA were determined using a Nanodrop spectrophotometer (Thermo Fisher Scientific, Wilmington, DE, USA). Subsequently, cDNA samples were synthesized using a High-Capacity cDNA Reverse Transcription kit (Applied Biosystems, Carlsbad, CA, USA) according to the manufacturer's instructions.

\section{RNA analysis and cecal bacteria analysis by qRT-PCR}

Gene expression levels of inflammatory cytokines (interleukin-8, IL-8; interferon- - , IFN- $;$; tumor necrosis factor- $a$, TNF- $a$; and interleukin-10, IL-10) and tight junction proteins (zonula occludens-1, ZO-1; occludin, OCLD; and claudin 1, CLDN1) in jejunal samples were determined by qRT-PCR using a RealHelixTM Premier qPCR kit (NanoHelix, Daejeon, Korea) with a StepOnePlus Real-Time PCR System (Applied Biosystems). Additionally, the expression levels of mucin 2 (MUC2) were also determined in both the jejunum and cecum. Glyceraldehyde-3-phosphate dehydrogenase (GAPDH) was used as a housekeeping gene. The $2^{-\triangle \Delta C T}$ method was used to quantify relative mRNA expression levels. The primers for the target genes are listed in Table 2 .

\section{Cecal gDNA extraction and quantification}

DNA was extracted using a DNeasyPowerSoil Kit (Qiagen, Hilden, Germany) according to the manufacturer's instructions. The extracted DNA was subsequently quantified using Quant-IT PicoGreen (Invitrogen).

\section{Library construction and Sequencing}

The sequencing libraries were prepared according to the Illumina 16S Metagenomic Sequencing Library protocols to amplify the V3 and V4 regions. The input gDNA, $2 \mathrm{ng}$, was PCR amplified using $5 \times$ reaction buffer, $1 \mathrm{mM}$ dNTP mix, $500 \mathrm{nM}$ each of the universal F/R PCR primers, and Herculase II fusion DNA polymerase (Agilent Technologies, Santa Clara, CA, USA). The cycling conditions for the first PCR were 3 min at $95^{\circ} \mathrm{C}$ for heat activation, and 25 cycles of $30 \mathrm{~s}$ at $95^{\circ} \mathrm{C}, 30 \mathrm{~s}$ at $55^{\circ} \mathrm{C}$, and $30 \mathrm{~s}$ at $72^{\circ} \mathrm{C}$, followed by a 5 min final extension at $72^{\circ} \mathrm{C}$. The universal primer pair with Illumina adapter overhang sequences used for the first amplification were as follows: V3-F: 5'-

TCGTCGGCAGCGTCAGATGTGTATAAGAGACAGCCTACGGGNGG CWGCAG-3', V4-R: 5'GTCTCGTGGGCTCGGAGATGTGTATAAGAGACAGGACTAC HVGGGTATCTAATCC-3'. The first PCR product was purified using AMPure beads (Agencourt Bioscience, Beverly, MA, USA). Following purification, $2 \mu \mathrm{L}$ of the first PCR product was PCR amplified for final library construction containing the index using NexteraXT Indexed Primer. The cycling conditions of the second PCR were the same as those for the first PCR, except those 10 cycles were run. Thereafter, the PCR product was purified using AMPure beads. The final purified product was then quantified using qPCR according to the qPCR Quantification Protocol 
Guide (KAPA Library Quantification kits for Illumina Sequencing platforms) and qualified using the TapeStation D1000 ScreenTape (Agilent Technologies Deutschland GmbH, Waldbronn, Germany). Pairedend $(2 \times 300 \mathrm{bp})$ sequencing was performed at Macrogen using the MiSeq ${ }^{\mathrm{TM}}$ platform (Illumina, San Diego, CA, USA).

\section{Cecal short-chain fatty acids (SCFA) measurement}

The concentration of short-chain fatty acids (SCFA) in the cecal contents was determined by gas chromatography-mass spectrometry (GC-MS). Briefly, $10 \mathrm{mg}$ of cecal contents were homogenized with an extraction solution consisting of $100 \mu \mathrm{L}$ of internal standard (100 $\mu \mathrm{mol} / \mathrm{L}$ crotonic acid), $100 \mu \mathrm{L}$ hydrochloric acid, and $200 \mu \mathrm{L}$ ether. After vigorous vortexing for $10 \mathrm{~min}$, the homogenates were centrifuged at $1,000 \times g$ for $10 \mathrm{~min}$, and $80 \mu \mathrm{L}$ of the supernatant was transferred into new glass vials. Aliquots were mixed with $16 \mu \mathrm{L}$ of $\mathrm{N}$-tert-butyldimethylsilyl-N-methyltrifluoroacetamide, and the vials were sealed tightly. The glass vials were heated at $80^{\circ} \mathrm{C}$ for $20 \mathrm{~min}$ in a water bath and then left at room temperature for $48 \mathrm{~h}$ for derivatization. The derivatized samples were run through a $6890 \mathrm{~N}$ Network GC System with an HP-5MS column and 5973N network mass selective detector (Agilent Technologies Deutschland $\mathrm{GmbH}$ ). Pure helium was used as the carrier gas delivered at a $1.2 \mathrm{~mL} / \mathrm{min}$ flow rate. The head pressure was set to $97 \mathrm{kPa}$ with a 20:1 split. The inlet and transfer line temperatures were 250 and $260^{\circ} \mathrm{C}$, respectively. The temperature program was as follows: $60^{\circ} \mathrm{C}$ for $3 \mathrm{~min}, 60-120^{\circ} \mathrm{C}\left(5^{\circ} \mathrm{C} / \mathrm{min}\right)$, and $120-300^{\circ} \mathrm{C}\left(20^{\circ} \mathrm{C} / \mathrm{min}\right)$. The run time was $30 \mathrm{~min}$, and the SCFA concentrations were quantified by comparing their peak areas with the standards.

\section{Statistical Analysis}

Growth performance was analyzed using the GLM procedures of SAS (1989), and polynomial contrasts (linear, quadratic, and cubic) were used to test the effect of SPL supplementation levels. The other physiological parameters (serum biochemical markers, gut histological data, gene expression levels in the jejunum and cecum, gut microbial population, and cecal SCFA concentrations) were analyzed using analysis of variance. All data analyses were conducted using SAS 9.4 (SAS Institute, Cary, NC, USA). Significant differences between the treatments were determined using Duncan's multiple range tests at a $p<0.05$ level of significance.

\section{Results}

\section{Growth performance}

At the end of the experiment, the piglets in the SPL5 and SPL10 treatment groups were 1.84 and $1.16 \%$ heavier, respectively, than those in the CON group, although the difference was not statistically significant $(p>0.05)$. During the experimental period, dietary SPL supplementation quadratically increased the ADG of piglets fed with the experimental diets in a dose-dependent manner $(p<0.05)$. However, ADFI and FE of pigs fed the SPL-supplemented diet did not differ among the treatments.

\section{Serum biochemical analysis}


The concentrations of glucose, triglyceride, total cholesterol, blood urea nitrogen, and creatinine were not changed by dietary SPL supplementation (Table 4). However, the albumin concentration of piglets in the CON group was significantly higher than that for piglets in the dietary SPL-supplemented groups $(p<$ 0.05).

Table 4

Biochemical markers of weanling pigs fed experimental diets

\begin{tabular}{|c|c|c|c|c|c|c|}
\hline & \multicolumn{6}{|c|}{ Dietary SPL levels (ppm) } \\
\hline & 0 & 5 & 10 & 15 & SEM $^{1}$ & p-value \\
\hline \multicolumn{7}{|c|}{ Serum biochemical markers, mg/dl } \\
\hline Glucose & 67.08 & 66.59 & 68.50 & 65.92 & 3.588 & 0.997 \\
\hline Triglyceride & 186.14 & 180.38 & 176.63 & 178.93 & 8.755 & 0.988 \\
\hline Total cholesterol & 86.47 & 85.27 & 83.95 & 83.11 & 1.470 & 0.886 \\
\hline Blood urea nitrogen & 5.22 & 5.02 & 5.12 & 4.44 & 0.331 & 0.863 \\
\hline Albumin & $4.22^{\mathrm{a}}$ & $3.84^{b}$ & $3.51^{b}$ & $3.80^{b}$ & 0.078 & 0.005 \\
\hline Creatinine & 0.82 & 0.81 & 0.81 & 0.84 & 0.006 & 0.162 \\
\hline
\end{tabular}

\section{Gut histological analysis}

Representative images of the jejunum stained by Alcian blue are presented in Fig. 1A. The SPL5 and SPL10 groups had significantly increased villus height, and piglets fed SPL-supplemented diets showed a significantly higher villus: crypt ratio compared with piglets in the CON group $(p<0.05$; Fig. 1B and 1C). Additionally, the number of goblet cells per villus height was significantly increased in the SPL10 treatment relative to the other treatments ( $p<0.05$; Fig. 1D).

\section{Gene expression levels in the gastrointestinal tract}

In the jejunum, dietary SPL supplementation significantly reduced the expression levels of proinflammatory cytokines IL-8, TNF- $a$, and IFN- $\gamma$ when compared to the CON group $(p<0.05)$ without modulation of the anti-inflammatory cytokine IL-10 (Fig. 2A). Additionally, the expression of genes related to tight junction proteins (ZO-1, OCLD, and CLDN) was significantly upregulated in the SPL5 and SPL10 groups when compared to the CON group ( $p<0.05$; Fig. $2 \mathrm{~B}$ ). Moreover, the expression level of MUC2 in the jejunum and cecum was significantly higher in the SPL5 group than those in the other treatment groups ( $p<0.05$; Fig. 2 C).

\section{The cecal microbial population at phylum and genus levels}


The Chao1 index of the gut microbiome was significantly increased in the SPL supplemented groups than in the CON group ( $p<0.05$; Fig. 3A); however, the Shannon and inverted Simpson indices were similar (Fig. 3B and 3C). Additionally, the microbial community of the SPL10 group was completely separated from that of the CON group (Fig. 3D).

The proportions of Bacteroidetes and Firmicutes at the phylum level were significantly higher and lower, respectively, in the SPL10 group relative to those of the CON group $(p<0.05 ;$ Fig. $4 \mathrm{~A})$. Furthermore, at the family level, the Prevotellaceae, Peptostreptococcaceae, and Parnesiellaceae populations were significantly higher in the SPL10 group than those in the CON group $(p<0.05 ;$ Fig. 4B). Moreover, the proportions of Prevotella, Gemmiger, Barnesiella, Parabacteroides, Phascolarctobacterium, and Alloprevotella were significantly upregulated in the SPL10 group than in the CON group ( $p<0.05$; Fig. 4 C).

\section{Cecal short-chain fatty acids (SCFA) concentration}

All SCFA types (acetate, propionate, and butyrate) were significantly increased in the SPL5 group compared with those in the CON group $(p<0.05$; Fig. 5$)$.

\section{Discussion}

Previous studies have demonstrated that dietary SPL could enhance intestinal wound healing capacity by upregulating mucus production in cell, rat, and poultry models [16-17]. MUC2 is the core protein of the membrane-linked mucus layer, and recent studies have demonstrated that MUC2 plays an important role in the gut defense system by lowering inflammation and improving gut integrity [18]. In addition, dietary SPL supplementation could increase the expression levels of jejunal and cecal MUC2 and the number of goblet cells in piglets. An improved gut mucus layer could protect against weaning stresses, including growth retardation and diarrhea.

Prevotella is the most dominant genus in the gastrointestinal tract of pigs, and its population gradually increases after weaning [19]. Moreover, Wright et al. demonstrated that Prevotella could utilize the mucus layer due to its mucin-desulfating glycosidase production [20]. Thus, the present study results suggest that dietary SPL supplementation could enhance the mucus layer, which would, in turn, dramatically increase the abundance of Prevotella after weaning. Moreover, the gut microbial complexity of animals considerably increases as they grow after weaning [21]. In the present study, dietary SPL supplementation increased the microbial richness and Chao 1 indices; notably, the SPL10 treatment group had a completely separate microbial community relative to the CON group. Altogether, our study demonstrated that optimal SPL supplementation in pig feed could improve the gastrointestinal defense system by reinforcing mucus thickness, resulting in an increase in the Prevotella population in piglet gut microbiota.

In the present study, piglets consumed similar amounts of feed, and the feed efficiency was also similar during the entire experimental period. However, the overall ADG of pigs fed with the SPL-supplemented diet increased quadratically in a dose-dependent manner. Our previous study with early-weaned rats demonstrated that dietary SPL supplementation $(10 \mathrm{mg} / \mathrm{kg})$ could accelerate rat growth after early 
weaning by improving the gut remodeling potential [16], consistent with the present study. On the other hand, Li et al. suggested that weaning stress could agitate the intestinal microbial population, resulting in a significant reduction in Bacteroidetes, as well as sharp declines in Prevotellaceae, particularly Prevotella populations [22]. Similarly, a comparable microbial shift at weaning was also found in the present study, with the SPL10 treatment significantly increasing the proportion of the Prevotella population. Additionally, March et al. reported that Prevotella-enriched pigs exhibited increased growth performance, which might be due to the ability of Prevotella to ferment complex polysaccharides in feed [23]. Here, we propose that dietary supplementation with $10 \mathrm{mg} / \mathrm{kg}$ SPL could improve gut bacterial modulation, which could increase ADG.

Albumin is a protein made by the liver that helps maintain fluid in the blood of animals by acting as a transport protein that binds to various ligands [24]. Therefore, a high serum albumin concentration could reflect the dehydration status of an animal and might indicate severe diarrhea [25]. In the present study, we found a significantly lower concentration of albumin in the sera of piglets fed with the SPLsupplemented diet than that of pigs in the CON group-the albumin concentrations of pigs in the SPL groups were within the normal range. This result indicated that dietary SPL could prevent dehydration due to weaning diarrhea by strengthening the gut defense system, a possible consequence of the dramatic increase in Prevotella abundance. Consistent with our study, various studies demonstrated that a higher abundance of intestinal Prevotella populations might have a protective effect against weaning diarrhea [26-27]. Thus, dietary SPL supplementation could improve gut resilience by ameliorating the immune response, strengthening tight junctions, and fortifying mucus production.

Decreased expression levels of pro-inflammatory cytokines without regulation of anti-inflammatory cytokines demonstrated that dietary SPL supplementation in the post-weaning period could downregulate the local immune response against external stresses. This phenomenon suggests that the dramatic increase in the Gemmiger population might potentially protect against inflammation after weaning. Forbes et al. demonstrated that the population of Gemmiger was significantly lower in patients with inflammatory bowel disease and consistently enriched in healthy people [28]. Moreover, Gemmiger spp. are among the candidate microbes in probiotic formulations for colorectal cancer [29]. Therefore, these results indicate that the addition of SPL to pig feed could ameliorate the immune response in the gut by increasing Gemmiger abundance in the intestinal microbial community.

Zhang et al. demonstrated that an increase in propionate in the hindgut could contribute to intestinal development and enhancement of jejunal barrier integrity [30]. Furthermore, the increase in butyrate concentration in the large intestine could not only enhance animal growth but also improve the intestinal mucosal environment in weaned piglets and growing pigs [31-32]. This might be due to the use of butyric acid as the main energy fuel for enterocytes in the large intestine [33]. In our study, optimal dietary SPL addition could increase the concentration of all SCFAs by increasing the Prevotella population in the cecum. Various studies have demonstrated that Prevotella showed high positive correlations with acetate, propionate, and butyrate production [34-36]. Moreover, Diao et al. proposed that increased gastric infusion of SCFA could decrease apoptosis in enterocytes by improving gut barrier function in 
weaned piglets [37]. Collectively, these results suggest that dietary SPL supplementation could aid the infusion of SCFAs into enterocytes and upregulate the restoration of gut integrity after weaning.

\section{Conclusions}

Sophorolipid supplementation in pig feed could control the gut microbiota population by improving the mucus layer. In addition, the upregulated infusion of SCFAs into enterocytes could strengthen tight junctions, increase mucin secretion capacity, and ameliorate immune responses. Our study indicated that $10 \mathrm{mg} / \mathrm{kg}$ SPL could be used as a feed additive in the post-weaning period of piglets for quick restoration of intestinal barrier function and integrity.

\section{DECLARATIONS}

\section{Declarations}

\section{Acknowledgements}

Not applicable.

\section{Funding}

This work was financially supported by EASYBIO Inc. and Korea University.

\section{Availability of data and materials}

Not applicable.

\section{Authors' contributions}

MJK and KYW conceived and designed the experiments; MJK and YSC mainly performed the experiments; MJK, YSC, and SWC analyzed the data; HBL and IGC contributed reagents/materials/analysis tools; MJK and SWC wrote the manuscript. All authors read and approved the final manuscript.

\section{Ethics approval}

All of works related to animal was conducted in accordance with the guidelines and regulations for the care and the use of experimental animals was approved by the Korea University Institutional Animal Care \& Use Committee (Permission No. KUIACUC-2021-0021)

\section{Consent for publication}

Not applicable.

\section{Conflicts of Interest}


The authors declare that they have no conflict of interest

\section{References}

1. Wu G, Meier SA, Knabe DA. Dietary glutamine supplementation prevents jejunal atrophy in weaned pigs. J Nutr. 1996;126:2578-84.

2. Neuenschwander S, Schwartz A, Wood TL, Roberts CT, Henninghausen L. Involution of the lactating mammary gland is inhibited by the IGF system in a transgenic mouse model. J Clin Invest. 1996;97:2225-32.

3. Danzeisen JL, Kim HB, Isaacson RE, Tu ZJ, Johnson TJ. Modulations of the chicken cecal microbiome and metagenome in response to anticoccidial and growth promoter treatment. PloS one. 2011;6:e27949.

4. Munk P, Knudsen BE, Lukjacenko O, Duarte ASR, Luiken RE, Van Gompel L, et al. Abundance and diversity of the fecal resistome in slaughter pigs and broilers in nine European countries. Nature. 2017;3:898-908.

5. De Lange CFM, Pluske J, Gong J, Nyachoti CM. Strategic use of feed ingredients and feed additives to stimulate gut health and development in young pigs. Livest Sci. 2010;134;124 - 34 .

6. Heo JM, Opapepju FO, Pluske JR, Kim JC, Hampson DJ, Nyachoti CM. Gastrointestinal health and function in weaned pigs: a review of feeding strategies to control post-weaning diarrhoea without using in-feed antimicrobial compounds. J Anim Physiol Anim Nutr. 2013;97;207 - 37.

7. Wang JP, Lee JH, Yoo JS, Cho JH, Kim HJ, Kim IH. Effects of phenyllactic acid on growth performance, intestinal microbiota, relative organ weight, blood characteristics, and meat quality of broiler chicks. Poult Sci. 2010;89:1549-55.

8. Roy A, Haldar S, Mondal S, Ghosh TP. Effects of supplemental exogenous emulsifier on performance, nutrient metabolism, and serum lipid profile in broiler chickens. Vet Med Int. 2010;11:262604.

9. Chen C, Jung B, Kim WK. Effects of lysophospholipid on growth performance, carcass yield, intestinal development, and bone quality in broilers. Poult Sci. 2019;98:3902-23.

10. Develter DW, Lauryssen LM. Properties and industrial applications of sophorolipids. Eur J Lipid Sci Techonol. 2010;112;628 - 38.

11. Desai JD, Banat IM. Microbial production of surfactants and their commercial potential. Microbiol Mol Biol Rev. 1997;61:47-64.

12. Finnerty WR. Biosurfactants in environmental biotechnology. Curr Opin Biotechnol. 1994;5:291-5.

13. Hardin R, Pierre J, Schulze R, Mueller CM, Fu SL, Wallner SR, et al. Sophorolipids improve sepsis survival: effects of dosing and derivatives. J Surg Res. 2007;142:314-9.

14. Chen J, Song X, Zhang H, Qu Y. Production, structure elucidation and anticancer properties of sophorolipid from Wickerhamiella domercqiae. Enzyme Microb Technol. 2006;39;501-6.

15. Otto RT, Daniel HJ, Pekin G, Müller-Decker K, Fürstenberger G, Reuss M, et al. Production of sophorolipids from whey. Appl Microbiol Biotechnol. 1999;52:495-501. 
16. Kwak MJ, Park MY, Kim J, Lee HB, Whang KY. Curative effects of sophorolipid on physical wounds: in vitro and in vivo studies. Vet Med Sci. 2021;7:1400-8.

17. Kwak MJ, Park MY, Choi YS, Cho J, Pathiraja D, Kim J, et al. Dietary sophorolipid accelerates growth by modulation of gut microbiota population and intestinal environments in broiler chickens. J Anim Sci Biotechnol. 2021;12:1-9.

18. Nishida K, Kamizato M, Kawai T, Masuda K, Takeo K, Teshima-Kondo S, et al. Interleukin-18 is a crucial determinant of vulnerability of the mouse rectum to psychosocial stress. FASEB J. 2009;23;1797 - 805 .

19. Holman DB, Brunelle BW, Trachsel J, Allen HK. Meta-analysis to define a core microbiota in the swine gut. mSystems. 2017;2;e00004-17.

20. Wright DP, Rosendale DI, Roberton AM. Prevotella enzmes involved in mucin oligosaccharide degradation and evidence for a small operon of genes expressed during growth on mucin. FEMS Microbiol Lett. 2000;190;73 - 9 .

21. Shao M, Wang Z, He Y, Tan Z, Zhang J. Fecal microbial composition and functional diversity of Wuzhishan pigs at different growth stages. AMB Express. 2021;11:1-9.

22. Li Y, Guo Y, Wen Z, Jiang $X, M a X$, Han $X$. Weaning stress perturbs gut microbiome and its metabolic profile in piglets. Sci Rep. 2018;8:1-12.

23. Mach N, Berri M, Estellé K, Levenez F, Lemonnier G, Denis C, et al. Early-life establishment of the swine gut microbiome and impact on host phenotypes. Environ Microbiol Rep. 2015;7;554 - 69.

24. Dong X, Zhang N, Zhou M, Tu Y, Deng K, Diao Q. Effects of dietary probiotics on growth performance, faecal microbiota and serum profiles in weaned piglets. Anim Prod Sci. 2014;54;616 - 21.

25. Okuma T, Nakamura M, Totake H, Fukunaga Y. Microbial contamination of enteral feeding formulas and diarrhea. Nutr. 2000;16;719 - 22.

26. Dou S, Gadonna-Widehem P, Rome V, Hamoudi D, Rhazi L, Lakhal L, et al. Characterisation of earlylife fecal microbiota in susceptible and healthy pigs to post-weaning diarrhoea. PLoS One. 2017;12;e0169851.

27. Sun J, Du L, Li X, Zhong H, Ding Y, Liu Z, et al. Identification of the core bacteria in rectums of diarrheic and non-diarrheic piglets. Sci Rep. 2019;9:1-10.

28. Forbes JD, Chen CY, Knox NC, Marrie RA, El-Gabalawy H, de Kievit T, et al. A comparative study of the gut microbiota in immune-mediated inflammatory disease - does a common dysbiosis exist? Microbiome. 2018;6:1-15.

29. Duvallet C, Gibbons SM, Gurry T, Irizarry RA, Alm EJ. Meta-analysis of gut microbiome studies identifies disease-specific and shared responses. Nat Commun. 2017;8:1-10.

30. Zhang Y, Chen H, Zhu W, Yu K. Cecal infusion of sodium propionate promotes intestinal development and jejunal barrier function in growing pigs. Animals. 2019;9; $284-93$.

31. Piva A, Morlacchini M, Casadei G, Gatta PP, Biagi G, Prandini A. Sodium butyrate improves growth performance of weaned piglets during the first period after weaning. Ital J Anim Sci. 2002;1:35-41. 
32. Gálfı P, Bokori J. Feeding trial in pigs with a diet containing sodium n-butyrate. Acta Vet Hung. 1990;38:3-17.

33. Roediger WE. Role of anaerobic bacteria in the metabolic welfare of the colonic mucosa in man. Gut. 1980;21:793-8.

34. Kishimoto A, Ushida K, Phillips GO, Ogasawara T, Sasaki Y. Identification of intestinal bacteria responsible for fermentation of gum Arabic in pig model. Curr Microbiol. 2006;53;173-7.

35. Wu Q, Liu W, Chen H, Yin Y, Hongwei DY, Wang X, et al. Fermentation properties of isomaltooligosaccharides are affected by human fecal enterotypes. Anaerobe. 2017;48; $206-14$.

36. Liang D, Li N, Dai X, Zhang H, Hu H. Effects of different types of potato resistant starches on intestinal microbiota and short-chain fatty acids under in vitro fermentation. Int J Food Sci. 2021;56:2432-42.

37. Diao H, Jiao AR, Yu B, Mao XB, Chen DW. Gastric infusion of short-chain fatty acids can improve intestinal barrier function in weaned piglets. Genes Nutr. 2019;14:1-16.

38. Xiao D, Tang Z, Yin Y, Zhang B, Hu X, Feng Z, et al. Effects of dietary administering chitosan on growth performance, jejunal morphology, jejunal mucosal slgA, occluding, claudin-1 and TLR4 expression in weaned piglets challenged by enterotoxigenic Escherichia coli. Int Immunopharmacol. 2013;17;670-6.

39. Cho WS, Chae C. Expression of Inflammatory Cytokines (TNF-aa, IL-1, IL-6 and IL-8) in Colon of Pigs Naturally Infected with Salmonella typhimurium and S. choleraesuis. J Vet Med. 2003;50;484-7.

40. Choi C, Cho WS, Kim B, Chae C. Expression of interferon-gamma and tumour necrosis factor-alpha in pigs experimentally infected with porcine reproductive and respiratory syndrome virus (PRRSV). J Comp Pathol. 2002;127;106 - 13.3.

41. Pié S, Lallès JP, Blazy F, Laffitte J, Sève B, Oswald IP. Weaning is associated with an upregulation of expression of inflammatory cytokines in the intestine of piglets. J Nutr. 2004;134:641-7.

42. Shang Q, Liu H, Liu S, He T, Piao X. Effects of dietary fiber sources during late gestation and lactation on sow performance, milk quality, and intestinal health in piglets. J Anim Sci. 2019;97:4922-33.

43. Limbach JR, Espinosa CD, Perez-Calvo E, Stein HH. Effect of dietary crude protein level on growth performance, blood characteristics, and indicators of intestinal health in weanling pigs. J Anim Sci. (2021);99;skab166.

44. Saqui-Salces M, Huang Z, Vila MF, Li J, Mielke JA, Urriola PE, et al. Modulation of intestinal cell differentiation in growing pigs is dependent on the fiber source in the diet. J Anim Sci. 2017;95:1179-90.

\section{Table 2}

Table 2. Oligonucleotide primers used in qRT-PCR analysis ${ }^{1}$ 
${ }^{1}$ Abbreviations: CLDN1, claudin-1; GAPDH, glyceraldehyde-3-phosphate dehydrogenase; IFN- $\gamma$, interferonY; IL-8, interleukin-8; IL-10, interleukin-10; MUC2, mucin 2; OCLD, occludin; TNF-a, tumor necrosis factor- a; ZO-1, zona occludens 1

\begin{tabular}{|c|c|c|}
\hline Gene name & Sequence (forward, reverse) & Reference \\
\hline \multicolumn{3}{|c|}{ Housekeeping gene } \\
\hline \multirow[t]{2}{*}{ GAPDH } & F: 5'- GAGGTCGGAGTGAACGGAT -3' & \multirow[t]{2}{*}{ [38] } \\
\hline & R: 5'- CCTGGGTCGAATCATACTGGAACA -3' & \\
\hline \multicolumn{3}{|c|}{ Inflammatory cytokines } \\
\hline \multirow[t]{2}{*}{ IL-8 } & F: 5'- TTTCTGCAGCTCTCTGTGAGG -3' & \multirow[t]{2}{*}{ [39] } \\
\hline & R: 5'- CTGCTGTTGTTGTTGCTTCTC -3' & \\
\hline \multirow[t]{2}{*}{ IFN-y } & F: 5'- GTTTTTCTGGCTCTTACTGC -3' & \multirow[t]{2}{*}{ [40] } \\
\hline & R: 5'- CTTCCGCTTTCTTAGGTTAG -3' & \\
\hline \multirow[t]{2}{*}{ TNF-a } & F: 5'- ATCGGCCCCCAGAAGGAAGAG -3' & \multirow[t]{2}{*}{ [41] } \\
\hline & R: 5'- GATGGCAGAGAGGAGGTTGAC -3' & \\
\hline \multirow[t]{2}{*}{ IL-10 } & F: 5'- GCATCCACTTCCCAACCA -3' & \multirow[t]{2}{*}{ [42] } \\
\hline & R: 5'- CTTCCTCATCTTCATCGTCAT -3' & \\
\hline
\end{tabular}

Tight junction proteins

\begin{tabular}{|c|c|c|}
\hline \multirow[t]{2}{*}{ ZO-1 } & F: 5'- AAGCCCTAAGTTCAATCACAATCT -3' & \multirow[t]{2}{*}{ [43] } \\
\hline & R: 5'- ATCAAACTCAGGAGGCGGC -3' & \\
\hline \multirow[t]{2}{*}{ OCLD } & F: 5'- TCCTGGGTGTGATGGTGTTC -3' & \multirow[t]{2}{*}{ [43] } \\
\hline & R: 5'- CGTAGAGTCCAGTCACCGCA -3' & \\
\hline \multirow[t]{2}{*}{ CLDN1 } & F: 5'- TCGACTCCTTGCTGAATCTG -3' & \multirow[t]{2}{*}{ [38] } \\
\hline & R: 5'- TTACCATACCTTGCTGTGGC -3' & \\
\hline \multicolumn{3}{|c|}{ Mucin production } \\
\hline \multirow[t]{2}{*}{ MUC2 } & F: 5'- GGCTGCTCATTGAGAGGAGT -3' & \multirow[t]{2}{*}{ [44] } \\
\hline & R: 5'- ATGTTCCCGAACTCCAAGG -3' & \\
\hline
\end{tabular}


(A)
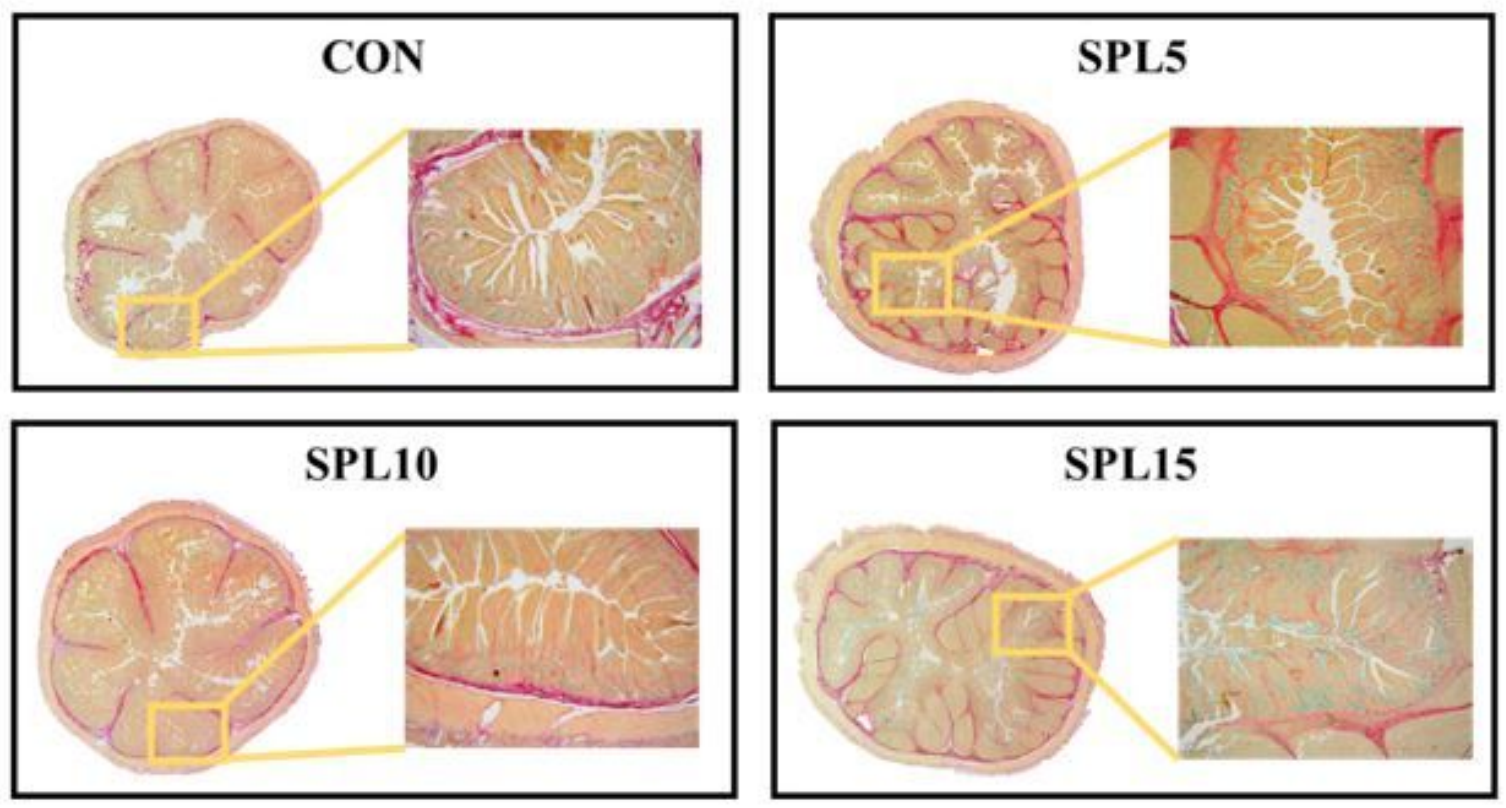

(B) Morphological data

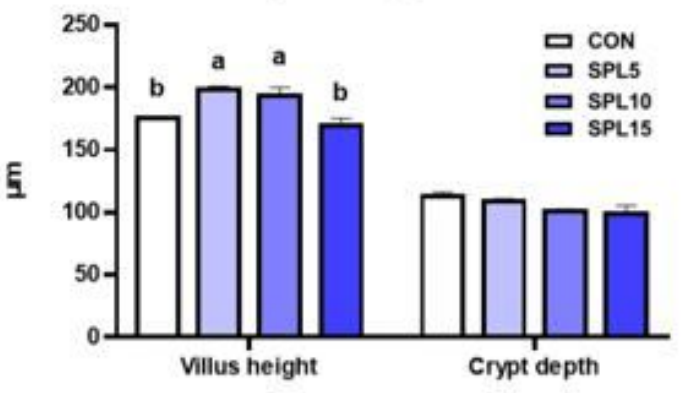

(C) Villus:crypt ratio

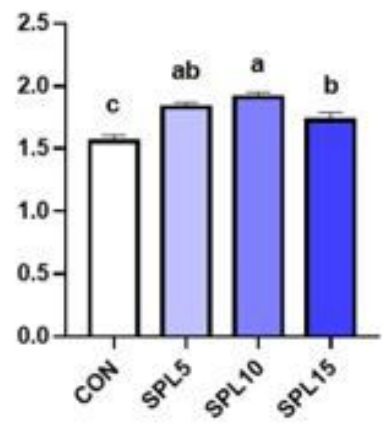

(D) Goblet cells / villus height

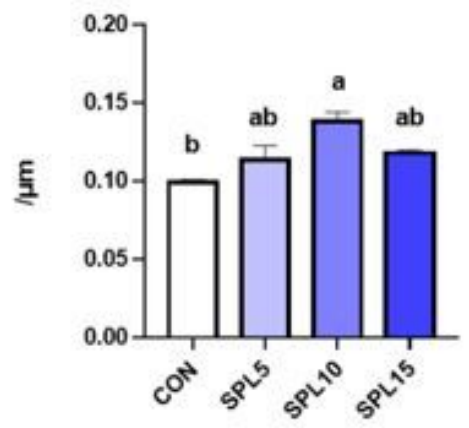

\section{Figure 1}

Gut morphological data from histological analysis of pigs fed experimental diets. A) Representative pictures of jejunum of pigs; B-C) Morphological indexes (villus height, crypt depth, and their ratio) of pigs;

D) The number of goblet cells per villus height in jejunum of pigs. a,b,c Mean values within a row have different superscript letters were significantly different $(P<0.05)$. Treatment groups: CON, control group fed with basal diet; SPL5, group fed with $5 \mathrm{mg} / \mathrm{kg}$ of SPL supplemented diet; SPL10, group fed with 10 $\mathrm{mg} / \mathrm{kg}$ of sophorolipid-supplemented diet; SPL15, group fed with $15 \mathrm{mg} / \mathrm{kg}$ of sophorolipidsupplemented diet. 


\section{(A) Inflammatory cytokines in jejunum}

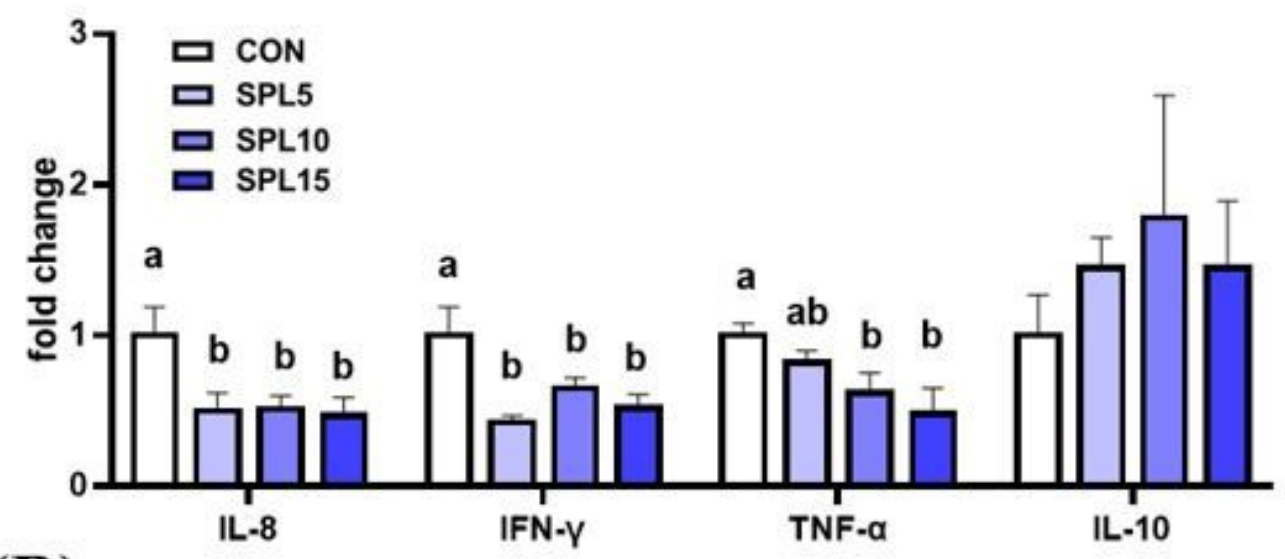

(B) Tight junction protein in jejunum

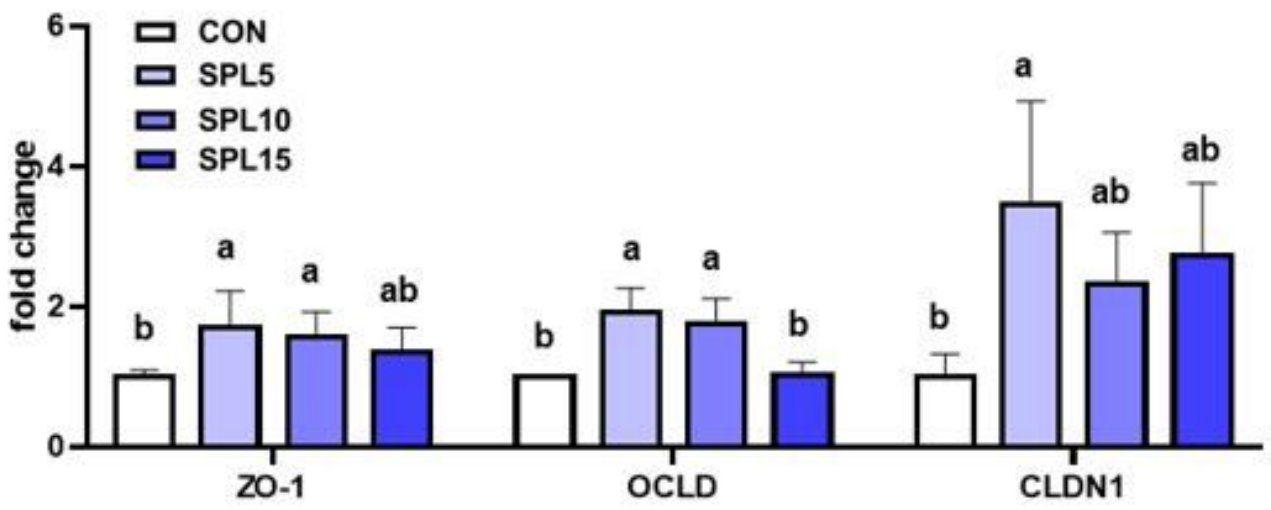

(C) Mucin 2

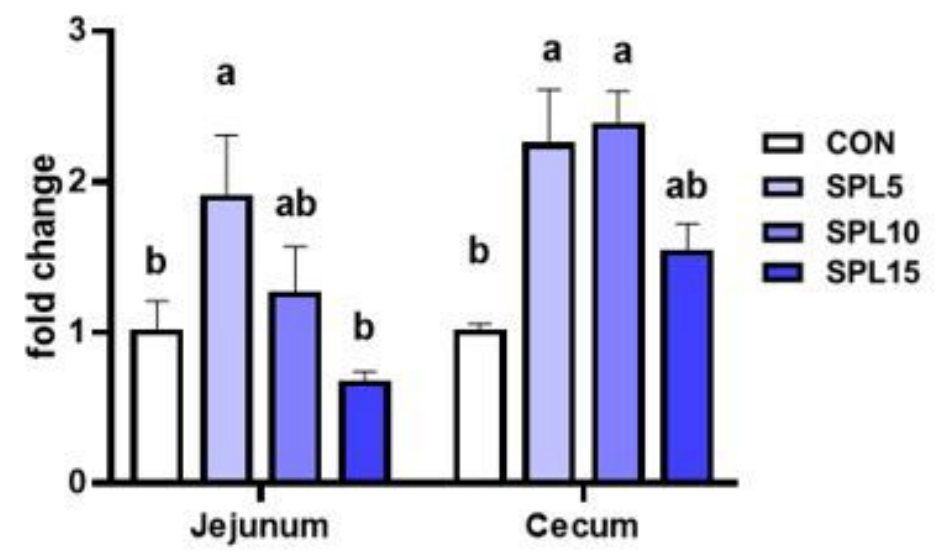

Figure 2

mRNA expression levels of genes in jejunum and cecum of pigs fed experimental diets. A) Genes related to inflammation cytokines (IL-8, IFN- $\gamma$, TNF-a, and IL-10); B) Genes related to tight junction proteins (ZO-1, OCLD, and CLDN1); C) MUC2 expression in jejunum and cecum. a,b Mean values within a row have different superscript letters were significantly different $(P<0.05)$. Treatment groups: CON, control group fed with basal diet; SPL5, group fed with $5 \mathrm{mg} / \mathrm{kg}$ of SPL supplemented diet; SPL10, group fed with 10 
$\mathrm{mg} / \mathrm{kg}$ of sophorolipid-supplemented diet; SPL15, group fed with $15 \mathrm{mg} / \mathrm{kg}$ of sophorolipidsupplemented diet. Abbreviations: IL-8, interleukin-8; IFN- $\gamma$, interferon- $\gamma$; TNF- $a$, tumor necrosis factor- $a$; IL-10, interleukin-10; ZO-1, zona occludensa-1; OCLD, occludin; CLDN1, claudin-1; MUC2, mucin 2.
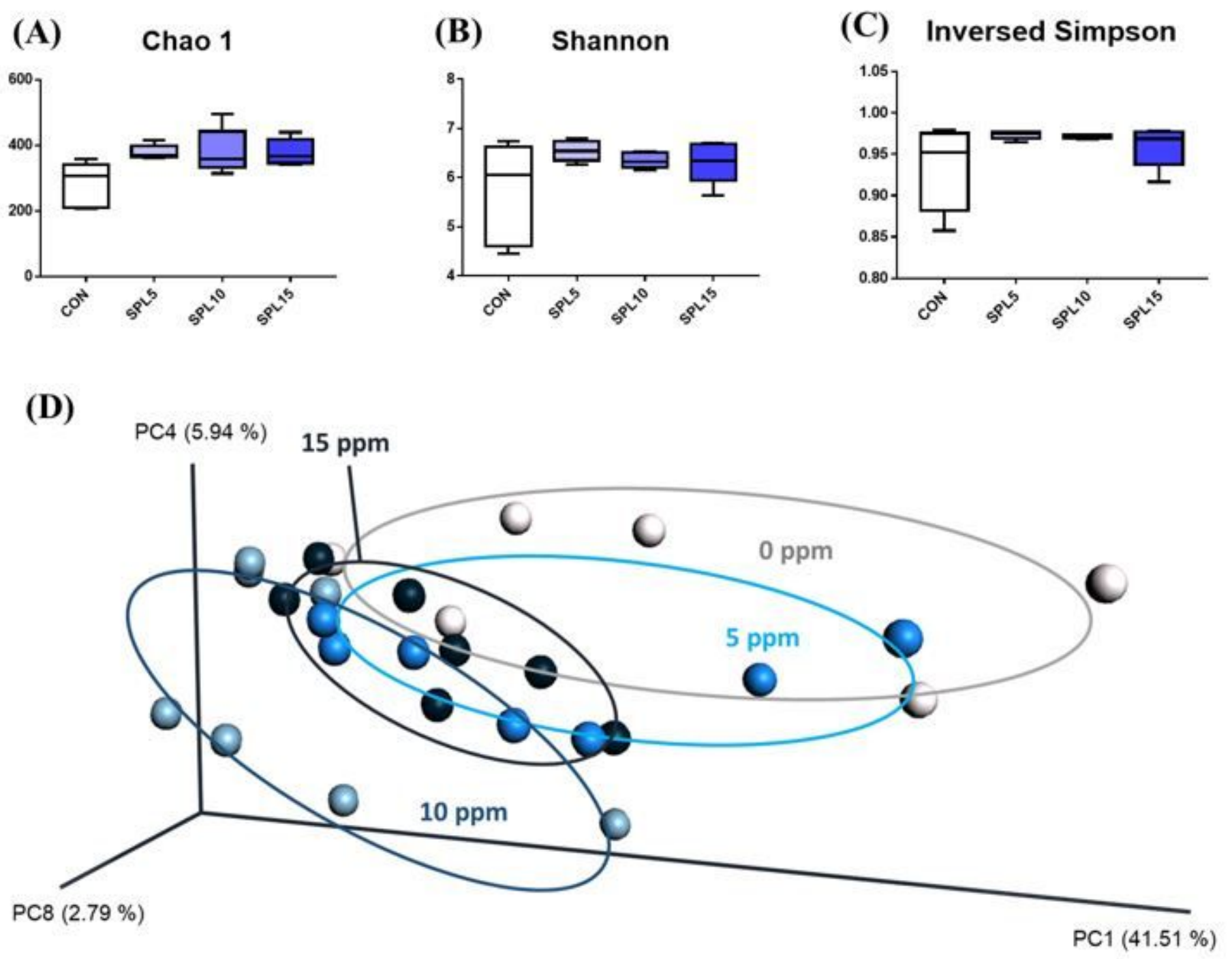

\section{Figure 3}

Cecal microbial community of broiler chickens fed experimental diets. A-C) Dietary effects of bambermycin and sophorolipid on species diverstiy indexes (Chao1, Shannon, and inversed simpson); D) Principal component Analysis ordination plots of microbial communities in the CON, SPL5, SPL10, and SPL15 groups based on the Jensen-Shannon distance metric. Treatment groups: CON, control group fed with basal diet; SPL5, group fed with $5 \mathrm{mg} / \mathrm{kg}$ of SPL supplemented diet; SPL10, group fed with 10 $\mathrm{mg} / \mathrm{kg}$ of sophorolipid-supplemented diet; SPL15, group fed with $15 \mathrm{mg} / \mathrm{kg}$ of sophorolipidsupplemented diet. 

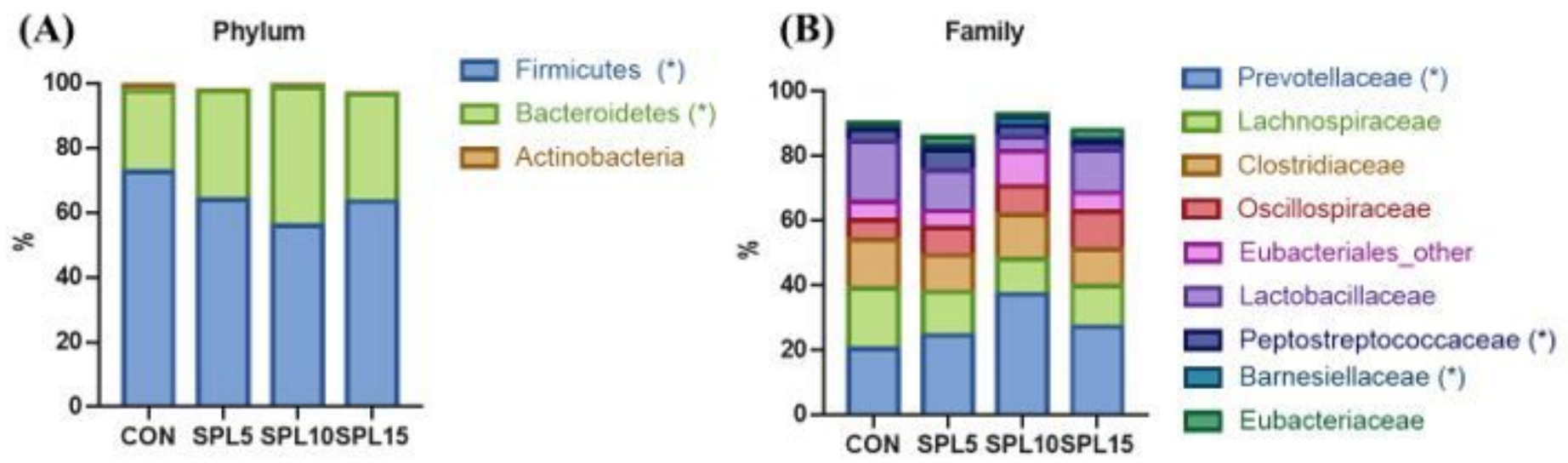

(C) Genus

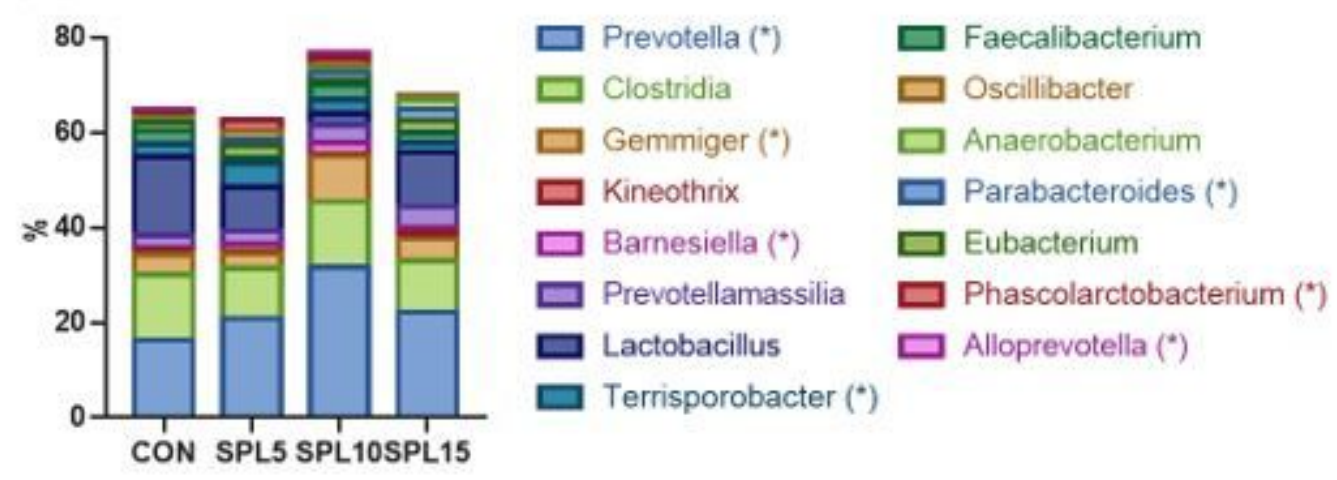

Figure 4

Gut microbiota population of pigs fed with experimental diets. A) Intestinal microflora at phylum level; B) Intestinal microflora at family level; $C$ ) Intestinal microflora at genus level. ${ }^{*} \mathrm{P}<0.05$ compared with $\mathrm{CON}$ group. Treatment groups: CON, control group fed with basal diet; SPL5, group fed with $5 \mathrm{mg} / \mathrm{kg}$ of SPL supplemented diet; SPL10, group fed with $10 \mathrm{mg} / \mathrm{kg}$ of sophorolipid-supplemented diet; SPL15, group fed with $15 \mathrm{mg} / \mathrm{kg}$ of sophorolipid-supplemented diet. 


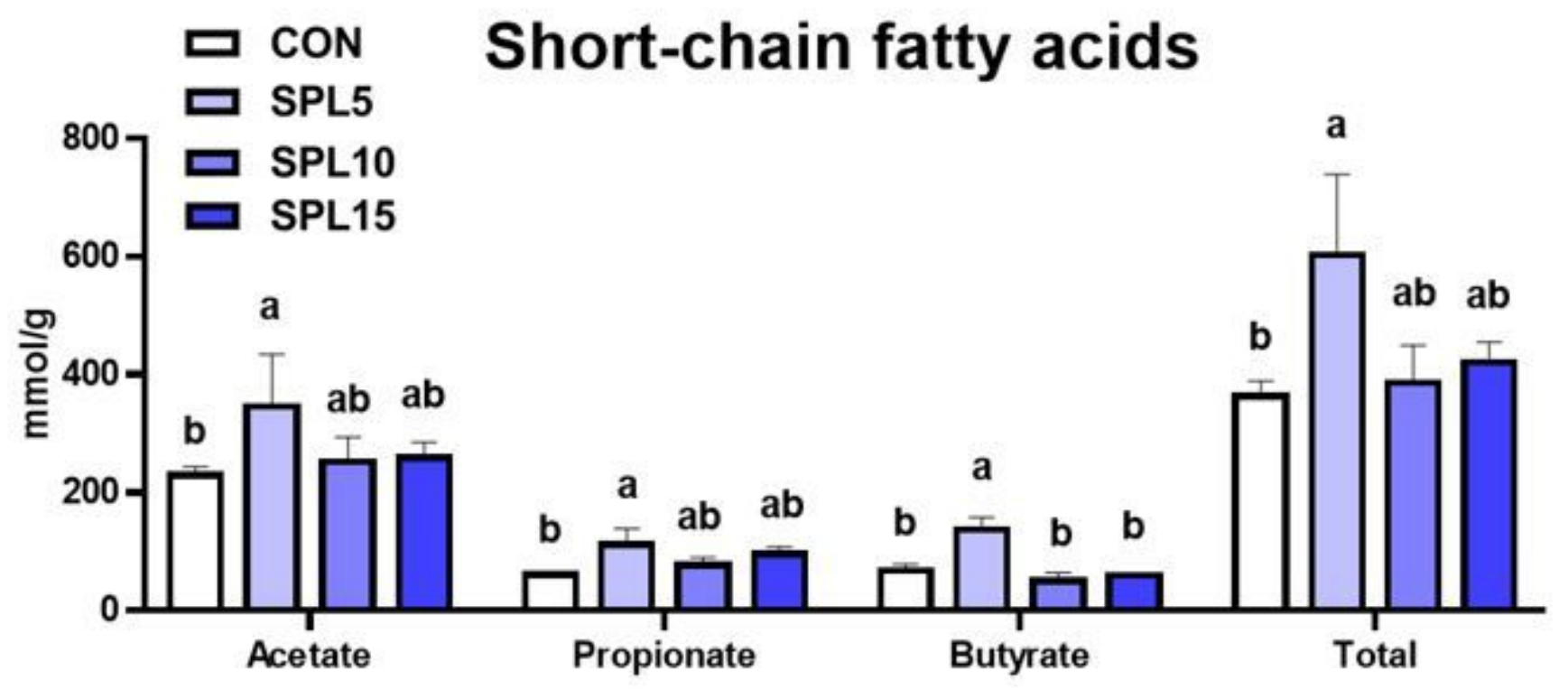

Figure 5

SCFA concentration (acetate, propionate, butyrate, and total) in cecum of pigs fed experimental diets. a,b Mean values within a row have different superscript letters were significantly different $(P<0.05)$. Treatment groups: CON, control group fed with basal diet; SPL5, group fed with $5 \mathrm{mg} / \mathrm{kg}$ of SPL supplemented diet; SPL10, group fed with $10 \mathrm{mg} / \mathrm{kg}$ of sophorolipid-supplemented diet; SPL15, group fed with $15 \mathrm{mg} / \mathrm{kg}$ of sophorolipid-supplemented diet. 